\title{
El maestro bibliotecario como agente propulsor de la biblioteca escolar
}

The teacher-librarian as a propellant agent of the school library

\author{
Carmen VARela Prado \\ Universidad de Santiago de Compostela \\ carmen.varela@usc.es
}

\begin{abstract}
Resumen
Se presenta una propuesta de formación del maestro bibliotecario, una posibilidad que se ofrece como itinerario formativo especializado en el grado de maestro en enseñanza primaria y como alternativa a la casi inexistencia de la figura del bibliotecario escolar en las escuelas españolas. Ante un entorno gris y contradictorio tanto social como educativo, presentamos a este posible futuro profesional como un agente propulsor de la biblioteca escolar y a esta como elemento estratégico para promover cambios en las metodologías de enseñanza/aprendizaje y actitudes más proactivas en estudiantes y profesores, más adaptadas a los retos a los que constantemente nos vemos sometidos en esta sociedad acelerada, tecnológica y global.
\end{abstract}

Palabras clave: Maestro-bibliotecario. Bibliotecarios escolares. Biblioteca escolar. Formación. España.

\section{Introduction}

El objetivo de este documento es exponer una propuesta para la formación del maestro bibliotecario, una opción posible y reconocida oficialmente en la Orden ECl/3857/2007 de 27 de diciembre (BOE, 2007) y que tomó forma en la memoria de verificación de la titulación de maestro en enseñanza primaria de la Facultad de Formación de Profesorado de Lugo. Esta iniciativa se presenta no con el optimismo y seguridad que debería acompañarla; en parte, por la coyuntura medioambiental de incertidumbre y desconfianza que está haciendo mella en las personas, mermando el empuje que necesitan algunas iniciativas que de por sí son difíciles de llevar a cabo.

No es solamente porque nos encontremos en una profunda crisis económica que afecta al desarrollo de cualquier proyecto por la minoración de medios, sino que - del análisis y reflexión sobre los diferentes entornos- llegamos a la conclusión de que nos movemos entre las buenas intenciones recogidas en textos normativos, declaraciones y manifiestos, pero que la realidad es otra, una contradicción que hace que esta propuesta pueda resultar una utopía.

\begin{abstract}
A proposal for teacher-librarian training is presented, a possibility that is offered as a specialized training route for future primary-school teachers and as an alternative to the absence of librarians in charge of the Spanish school libraries. Facing a gray and contradictory environment, both socially and educationally, we present this prospective future professional as a propellant of school libraries and as a strategic factor to promote changes in the methodologies of teaching-learning and to stimulate proactive attitudes in students and teachers, so they become more adapted to the challenges that we are constantly undergoing in this accelerated, technological and global society.
\end{abstract}

Keywords: Teacher-librarian. School libraries. School librarians. Professional education. Spain.

El maestro bibliotecario como profesional que lidere un modelo de biblioteca escolar como espacio de aprendizaje abierto, que sea agente promotor de metodologías de aprendizaje constructivistas y activas, que contribuya a que estos pequeños estudiantes generen desde el inicio de sus estudios capacidades de creación, de interacción y autonomía, y que al mismo tiempo, interactúen con sus colegas para establecer objetivos comunes, puede sonar a fantasía. Sin embargo, creemos en esta posibilidad. No obstante, tendrán que ser profesionales con los conocimientos y habilidades apropiadas, además de estar dotados de una buena carga vocacional, de convencimiento y una gran dosis de generosidad.

Es verdad que la literatura específica sobre biblioteca escolar, producto de la investigación de expertos, se mueve en esta dirección; que las buenas prácticas que se conocen, significan claros ejemplos desarrollados por profesionales con dedicación e implicación, en gran parte gratuita y altruista; y que los múltiples productos u objetos de aprendizajes difundidos en la red son exponentes de avance. Pero no existe una política generalizada y reglada, ni lazos de co- 
nexión, salvo en los encuentros, jornadas y redes específicas de trabajo, en las que se comparten las distintas experiencias y se aúnan elementos de motivación.

Si el objeto de la biblioteca escolar y la actuación de los profesionales son los escolares, es necesario analizar los distintos escenarios en los que nos movemos. Son los marcos en los que tenemos que encuadrar a nuestra actual infancia, a los maestros y a los bibliotecarios: marco social, educativo y profesional. Analizar cuál es el entorno social actual, lo que les estamos ofreciendo a los jóvenes estudiantes desde las instituciones educativas, que necesitan y van a necesitar como adultos, como ciudadanos y profesionales con la responsabilidad de construir el futuro, un futuro que ya parte de un presente envuelto en una revolución continua y sucesiva de cambios y de incertidumbre.

Se necesita realizar un análisis psicosociológico y de desarrollo de los niños/as de nuestros tiempos, en un entorno que no es comparable al que crecieron sus padres, abuelos y maestros. Esto implica, un esfuerzo de adaptación a los tiempos por parte de progenitores y profesores para entender muchas de sus actitudes, hábitos y comportamientos, buscando fórmulas que propicien el entendimiento y acercamiento, para que no se produzca una ruptura generacional y pueda fluir la interconexión positiva de experiencias entre generaciones.

Con todo esto, intentamos diseñar el marco formativo que permita obtener un perfil de maestro bibliotecario que reúna los conocimientos específicos de su titulación, las habilidades pedagógicas que le han de acompañar y una formación específica como bibliotecario, entendiéndolo no como el gestor de un espacio con libros y materiales que ha de organizar, sino con una formación transversal plasmada en los contenidos de las materias específicas $y$, sobre todo, con la utilización de una metodología de aprendizaje basada en proyectos, que contribuya a que también adquiera una actitud para su trabajo como maestro, que pueda aplicar metodologías basadas en el constructivismo, la interactividad en "aprender haciendo", para que los niños/as desde la primeras etapas de su infancia activen las mentes y despierten sentidos naturales como la curiosidad, querer descubrir, reflexionar, contextualizar, relacionar, analizar, manifestar sus opiniones, crear y ser autónomos... En fin, empezar a saber ser, estar y hacer.

\section{2. ¿Que se dice de la biblioteca escolar? Un repaso a la literatura, las buenas prácticas y lasredes de trabajo colaborativo}

En primer lugar es imprescindible citar la recientemente desaparecida publicación Educación y biblioteca, uno de los principales medios de expresión y comunicación que Francisco J. Bernal fundó con el objetivo de difundir y al mismo tiempo contribuir a superar la marginación educativa de la biblioteca (Bernal, 1989). Y quien mejor ha fotografiado la situación de la biblioteca escolar ha sido precisamente Jose Antonio Gómez Hernández (2010) en un informe publicado en el Anuario Thinkepi 2010. En este informe, se ofrece un estado de la cuestión amplio y detallado que refleja la situación precaria de la biblioteca escolar en nuestro país, así como los avances que se han ido produciendo, representados por las referencias a buenas prácticas y los productos y acciones de redes colaborativas. De este informe, que he tomado como fuente de información bibliográfica, hemos tenido en cuenta aquellos documentos y autores/as que nos han supuesto un aval para afianzar nuestras reflexiones y fortalecer la propuesta que se presenta del maestro con formación bibliotecaria.

El estudio global sobre la situación de la biblioteca escolar realizado por Marchesi y Miret en el año 2005 demuestra su existencia como espacio depósito de libros, sin asistencia profesional y menos como elemento integrado en las funciones docentes de los centros escolares. Nada que ver, por ejemplo, con la situación en Francia, donde ya a finales de los 80 , se aprueba que todo centro docente tenga un centro de documentación e información, así como un titulado especialista en su gestión y uso educativo (Gómez-Hernández, 2010).

Han pasado 8 años desde la publicación del informe y reflexiones de Miret y no hemos avanzado en gran medida o en la línea que se debía al amparo de las instituciones educativas; y peor pinta el panorama, amenazado por la influencia devastadora de la crisis.

Habiendo hecho una pequeña cata por los Centros de Educación Infantil y Primaria (CEIP) de nuestra ciudad, las comprobaciones siguen coincidiendo con las reflexiones de Miret. Si además añadimos que - según los resultados que estamos obteniendo de las encuestas en los cursos de iniciación en competencias informacionales al acceder a la Universidad (estudiantes que proceden de la secundaria)-, la media ponderada, entre 0 y 5 , de los alumnos/as que han recibido formación, han usado la biblioteca de su centro, saben organizar un tra- 
bajo o saben cómo utilizar la información, no llega a 3 , ¿qué vamos a pensar de los centro de infantil y primaria?

Las bibliotecas en las escuelas siguen siendo espacios dotados de materiales que llegan fundamentalmente por dotación de las consejerías de educación de las comunidades autónomas y cada vez menos, en los que en ocasiones existe algún tipo de servicio de préstamo, a los que los estudiantes acuden para realizar su "hora de biblioteca", cuya misión es el fomento de la lectura literaria, que el profesorado no utiliza como fuente de información, y dónde en ocasiones se realizan algunas actividades promocionales 0 algún tipo de exposición conmemorativa. En conclusión, en nuestras escuelas existe ese espacio fantasma, en el mejor de los caso con cierta organización, que carecen de profesionales, porque la biblioteca escolar no es un nicho laboral incluido en la carrera profesional; y los brotes emergentes surgen gracias a la implicación y esfuerzo de algún maestro que ha entendido la función que debe de desempeñar la biblioteca en su centro escolar: No olvidemos, que lo importante son siempre las personas y sus actitudes y no los espacios.

Coincidimos plenamente con Gloria Durban en que, lo que justifica la existencia de la biblioteca escolar, no es la biblioteca como espacio, equipamiento y servicio bibliotecario, sino como recurso educativo útil para realizar en ella intervenciones didácticas, integrándose con las distintas materias del currículo, actuando en colaboración con sus colegas y juntos orientar a los alumnos/as persiguiendo objetivos comunes. Esto es, coincidimos en visualizar la biblioteca como centro catalizador de múltiples demandas de recursos educativos que puedan elaborar con eficacia el bibliotecario y el maestro (Durban, 2009).

Para los estudiantes, la biblioteca será un entorno que les facilitará un entrenamiento intelectual y competencial imprescindible, que contribuirá a que el escolar empiece a afianzar capacidades para su desarrollo personal y social.

La biblioteca ha de adquirir un posicionamiento en el entorno escolar, no solamente como un espacio físico, sino como eje propulsor de procesos que redunden en la mejora de la enseñanza y el aprendizaje del alumnado.

El reconocimiento de la biblioteca como eje catalizador solamente se podrá desarrollar con el apoyo continuado del equipo de dirección y la aceptación y colaboración del equipo docente, para realmente generar procesos de mejora y encontrar su anclaje en el sistema educativo como herramienta facilitadora y de apoyo a la labor docente.

No olvidemos que este eje ha de estar vinculado muy estrechamente a las nuevas tecnologías, una realidad imprescindible hoy día, vistas como herramientas que favorecen el desarrollo de los procesos de aprendizaje y metodologías de actuación (Durban, 2009):

La conclusión es clara, la clave de la biblioteca escolar no está en la biblioteconomía que aborda la organización y dinamización de un servicio bibliotecario, sino en la pedagogía y en la disciplina específica que abarca el estudio de la didáctica y la organización escolar, donde está incluida la tecnología educativa. Solo desde este ámbito conectaremos con la substancia educativa de la biblioteca escolar y su sentido, al definir y esclarecer su articulación como recurso educativo útil y como medio para el alcance de objetivos y metas educativas.

Necesitamos que nuestros alumnos/as lean y escriban para que aprendan a pensar. Esta es la gran necesidad. Hemos de movilizar sus anhelos de curiosidad crítica, su querer saber y conocer no solo para aprender, sino también, para encauzar y dar sentido así, a la acción creativa y transformadora que queremos que sean capaces de ejercitar.

Comparto con Gómez-Hernández que llegar a una simbiosis efectiva de biblioteca y educación está siendo una labor titánica; y cada vez más, pues a los logros que parecían haberse alcanzado, vemos como sobreviene un retroceso motivado por la crisis generalizada, pero también por el cansancio, la incertidumbre, la frustración que producen los sucesivos cambios legislativos en la educación: Todo ello influye que la motivación e ilusión merme. Sin embargo, es de justicia resaltar los documentos, acciones y buenas prácticas llevadas a cabo por grupos de trabajo colaborativo; y la celebración de encuentros y congresos que reúnen a profesionales que siguen en la lucha por visibilizar propuestas y logros alcanzados.

A nivel institucional encontramos ejemplos de reconocimiento y apoyo a las buenas prácticas y experiencias orientadas al impulso de la biblioteca escolar. Es importante visibilizar los éxitos, demostrar que son posibles y difundirlos para resaltar el protagonismo educativo de las bibliotecas escolares.

Citamos como ejemplo el apoyo del Centro Coordinador de Bibliotecas escolares de la Xunta de Galicia, y el portal que recoge todas las iniciativas que se llevan a cabo en la comunidad y que contribuye a la formación, organización, y difusión de buenas prácticas y la creación de espacios de encuentro como las Xornadas de bibliotecas escolares, que se celebran anual- 
mente, o Os encontros do Plan de mellora de bibliotecas escolares 2012/2013.

Así mismo ocurre en otras comunidades: Andalucía, Asturias, Cataluña, Extremadura, Murcia, Navarra (Gómez-Hernández, 2010)

Hay que destacar también la función que durante estos años ha desempeñado la Fundación German Sánchez Ruipérez en la organización de las Jornadas sobre bibliotecas infantiles, juveniles y escolares, ya en su vigésima edición; foro en el que se llevan planteados problemas, avances y novedades que afectan a las bibliotecas infantiles, juveniles y escolares.

También hay que tener en cuenta portales de ayuda para docentes y bibliotecarios como El canal lector sobre libros infantiles y juveniles recomendados (http://www.canallector.com/); Leer.es (http://www.leer.es), portal de apoyo para mejorar la comprensión lectora; el Portal Intef del Instituto Nacional de Tecnología Educativas y de Formación de Profesorado del Ministerio de Educación, cultura y Deportes, que presenta $A B I E S$, un programa de gestión de bibliotecas escolares en el que participan varias comunidades autónomas; o el modelo de biblioteca digital educativa y la propuesta metodológica para la creación en entornos escolares de la biblioteca escolar como Centro de Recursos para la enseñanza y el Aprendizaje (CREA) (Marzal y Sellers de los Rios, 2011).

Todas estas iniciativas son significativas y hacen posible mantener viva la biblioteca escolar y seguir apostando por ella como elemento estratégico en la educación.

\section{3. ¿En qué escenarios nos movemos? Económico, social, educativo, docente, profesional}

A largo de esta revisión bibliográfica, han quedado claros el modelo de biblioteca y el profesional que defendemos en el ámbito escolar, mucho argumento teórico y poca realidad pragmática, pero aun así, ¡creamos en la utopía realizable y justificable!

En primer lugar, escribamos los distintos escenarios y veremos las contradicciones que se dan entre unos y otros.

\subsection{Escenario económicosocial}

Nos encontramos en una nueva era que nos sobrevino con una aceleración incontrolable, debido al avance de la tecnologías de la información y comunicación; y que generó la llamada sociedad del conocimiento y el concepto y realidad de la globalización, una sociedad que, en la actualidad, se encuentra sometida y a merced del juego económico-financiero y bajo el dominio de intereses transnacionales dirigidos.

A esta sociedad - centrándonos en la europea y más concretamente en la española-le cuesta, con una edad media de 40,9 años, asumir los cambios que produce la propia aceleración a la que se ve sometida en todos los ámbitos, sobrecargada por el desbordante flujo de información, incapaz de poder procesar, mediatizada por los medios de comunicación y muchas veces perdida por la redes sociales. Se ve agobiada por la transformación que supone la incorporación de las nuevas tecnologías y procedimientos en sus vidas laborales; y cada vez es más escéptica con respecto a los poderes públicos. Pero esto no tiene vuelta atrás, y lo que está claro es que "el aprendizaje para toda la vida" es una lógica impensable hoy día. Cualquier ciudadano/a necesita adquirir una serie de competencias y habilidades para desenvolverse no solamente como profesional, sino también como persona; y no puede negar la evidencia, porque traerá consigo ignorancia consentida, apatía, resignación... y cada vez serán más variadas y profundas las brechas sociales.

Por todo ello, los futuros ciudadanos, que son los niños/as y jóvenes que ahora comienzan sus estudios en las escuelas y en las universidades, al amparo todavía de sus progenitores, necesitan aprender a desarrollar desde el principio competencias y capacidades de carácter transversal como son la comprensión lectora; la capacidad de análisis, de relación y contextualización; el espíritu crítico; la capacidad de cooperación, de autonomía, de autodisciplina, de trabajo, que se sumarán a los conocimientos específicos.

\subsection{Escenario educativo}

Ante un modelo de sociedad como el que se acaba de describir y que empieza a despuntar en los años 90 del siglo pasado, las instituciones y legisladores educativos adquieren la responsabilidad de modificar los sistemas y metodologías de enseñanza dándole nuevas orientaciones. En las sucesivas leyes de educación que se publicaron, tengamos en cuenta en primer lugar la que afecta al sistema educativo en general y en segundo lugar, la legislación propia de Universidades. Tanto los maestros como los bibliotecarios se forman en la universidad y están afectados por la legislación correspondiente; y como maestros y/o bibliotecarios escolares han de ajustarse a la legislación que les compete. 
En el recorrido que se hace a través de la legislación educativa, se intenta mostrar la filosofía de cada una de las leyes dictadas con respecto a la tipología de enseñanza y las metodologías recomendadas.

Si nos remontamos a la Ley General de Educación de 1970, ya aparece entre sus recomendaciones la de utilizar métodos de enseñanza activa, original y creativa.

Con la restauración democrática, se promulga la Ley Orgánica de Derecho a la Educación (LO$\mathrm{DE})$ de 1985 con una dirección abierta y democrática. La Ley Orgánica de Ordenación General del Sistema Educativo (LOGSE) de 1990 busca establecer una enseñanza basada en fundamentos educativos más técnicos y autónomos por parte del alumno. En dicha ley se reclaman aspectos como la consulta de fuentes de información como procedimiento de trabajo, el uso correcto del lenguaje, el desarrollo del espíritu crítico y se apuesta por un currículum abierto, por la adquisición de destrezas y actitudes, por un aprendizaje significativo en contraposición a los aprendizajes repetitivos y memorísticos, y lo más importante, que en la escuela se enseñe a "aprender a aprender" haciendo énfasis en la capacidad de aprender por sí mismos (Bueno Monreal, 1997).

En el año 2002 se publica la Ley Orgánica de Calidad de la Educación (LOCE) y entre sus objetivos está que los alumnos desarrollen valores y principios básicos de creatividad, iniciativa personal y espíritu emprendedor. Se promociona la investigación, la experimentación y la innovación educativa fomentando actitudes de confianza, sentido crítico, creatividad e iniciativa personal (BOE 307, 2002).

La ley Orgánica 2/2006, de 3 de mayo, de Educación (LOE) destaca entre sus objetivos el fomentar el aprendizaje a lo largo de toda la vida y proporcionar a los jóvenes una educación completa, que abarque conocimientos y competencias para su desarrollo personal y profesional; aprender a desarrollar hábitos de trabajo individual y de equipo, de esfuerzo y responsabilidad en el estudio; así como actitudes de confianza en sí mismo, sentido crítico, iniciativa personal, curiosidad, interés y creatividad en el aprendizaje. Esta ley regula la existencia de la biblioteca escolar en el art. 113. (BOE 106, 2006)

Por último está el Proyecto de Ley para la Mejora de la Calidad Educativa (LOMCE) de 2013, la séptima norma educativa en 37 años de democracia y pendiente de aprobación. Sin profundizar demasiado, de la exposición de motivos y la organización de las enseñanzas, se puede ex- tractar que es una ley tecnicista y dirigida a descubrir y potenciar talentos, que busca la competitividad económica, la empleabilidad, la enseñanza basada en conocimientos, recursos y herramientas - ¿donde están las competencias, los valores?-, las TIC son herramientas complementarias (no integradas), un sistema directivo y centralizado, un diseño pensado en clave de resultados y dirigido exclusivamente a poder cumplir con los valores de los indicadores de la Estrategia europea 2020 de la Unión Europea.

En la Universidad, partimos de la Ley de Reforma Universitaria (LRU) de 1983, primera ley después de la instauración de la democracia, que defiende la democratización de los estudios universitarios como la base más sólida para una sociedad tolerante, libre y responsable. Declara como funciones básicas de la universidad el desarrollo científico, la formación profesional y la extensión cultural. Defiende una universidad donde arraiguen el pensamiento libre, crítico y de investigación, instrumento eficaz de transformación social y al servicio de la igualdad y el progreso social (BOE 209, 1983).

A finales del siglo $X X$, la universidad española comienza a planear una transformación derivada de los acuerdos establecidos en la Declaración de Bolonia y la integración en el Espacio Europeo de Educación Superior y el Espacio Europeo de Investigación, un proceso que deberá estar totalmente implementado en el año 2010.

Se publica la Ley Orgánica de Universidades (LOU) en el 2001; y la filosofía de esta ley ya va teniendo presentes las exigencias de la nueva sociedad demandante de profesionales con elevado nivel cultural, científico y técnico, que exige una formación permanente a lo largo de la vida, no solo en el orden macroeconómico y estructural, sino también como modelo de autorrealización personal y donde se reconoce que la sociedad de la información necesita personas capaces de convertir la información en conocimiento mediante su ordenación, elaboración e interpretación (BOE 307, 2001).

En el año 2007 se publica la Ley Orgánica 4/2007 que modifica la Ley Orgánica 6/2001 y donde se estructuran la enseñanzas universitarias de acuerdo al modelo común europeo, que va a constituir una profunda reforma curricular basada en la flexibilidad, en la transversalidad y la multidisciplinaridad; $y$ donde la enseñanza/aprendizaje ha de medirse en términos de conocimientos y competencias que debe adquirir el estudiante. (BOE 89, 2007). 
Pues bien, estos son los marcos normativos que han afectado a la educación en España en los últimos años y que responden a los cambios que se han venido produciendo, principalmente por la adaptación a las directrices marcadas desde la Unión Europea en temas de educación y también debido a las políticas de los gobiernos de distinto signop que en ninguna de las etapas han conseguido establecer un pacto en cuanto a política educativa y sistemas que pudiesen perdurar en el tiempo. Podríamos añadir a esta revisión legislativa también decretos de modificación o desarrollo, libros blancos, legislación propia de las comunidades y los diferentes estatutos de las universidades, pero, en líneas generales, abundan en la misma dirección.

\subsection{Escenario docente-profesional}

Haciendo un repaso a toda la legislación presentada, recuperamos una serie de conceptos fundamentales que queremos enfatizar: "métodos de enseñanza activa, original y creativa", "enseñanza/aprendizaje" "desarrollo del espíritu crítico", "aprendizaje significativo", "aprender a aprender", "creatividad, iniciativa personal y espíritu emprendedor", "innovación educativa", "curiosidad, interés y creatividad, autorrealización personal", "convertir la información en conocimiento mediante su ordenación, elaboración e interpretación", "transversalidad y la multidisciplinaridad", "conocimientos y competencias".

¿Qué tanto de desarrollo tuvieron estas acciones en la realidad de la enseñanza tanto no universitaria como universitaria? Realmente, ¿fueron entendidos muchos de estos conceptos? Más bien no mucho, salvo en el área de la pedagogía y resucitando a los constructivistas, Jean Piaget, Lev Vygotski.

Tanto el maestro como el bibliotecario, adquieren su formación en la universidad. Analicemos pues, el entorno docente real: ¿El profesorado universitario ha incorporado en la organización de los programas de sus materias y en sus metodologías estos objetivos realmente? ¿Los docentes han reformulado el papel y la práctica pedagógica planteando modelos de aprendizaje distintos a los tradicionales? La realidad demuestra que los estudiantes siguen aprendiendo en un modelo de enseñanza de transmisión de conocimiento en el que el docente controla la información y sigue utilizando técnicas eminentemente expositivas, un modelo transmisivo de información elaborada y definitiva que el docente transfiere al alumnado y que este debe asumir sin cuestionarlo (Área, 2005; Varela-Prado, 2009). En el nuevo modelo "Bolonia" la enseñanza se organiza en clases expositivas, grupos interactivos y tutorías. Las clases expositivas siguen siendo las lecciones magistrales de siempre; las interactivas, para organizar y controlar trabajos académicos; y las tutorías, para resolver algún problema. Se han sustituido las fotocopiadoras por las plataformas, en las que el profesor cuelga contenidos que recuperan los alumnos y suben los trabajos: A eso le llamamos innovación. La evaluación, aunque continua, se resuelve al final con el examen de siempre y, cada vez más, tipo test. ¿Cómo se puede saber si el alumnado ha adquirido las competencias que cada profesor ha definido en la memoria de verificación de la titulación y en su guía docente? ¿Esta metodología da margen a que el alumno/a sea creativo, desarrolle un espíritu crítico...?

El espíritu de Bolonia no fue bien entendido y el modelo europeo fue implantado sin la pedagogía necesaria y el tiempo de asimilación del cambio. En este país estaba muy arraigada la enseñanza basada en el testbook, en la memorización y en metodologías directivas, muy distintas a las que ya estaban extendidas en el resto de los países europeos.

A estos problemas, hay que añadir la organización de los enseñanzas universitarias según un modelo "sui géneris" de grados de 4 años y oferta de master, frente a la ordenación europea de grados de 3 años y 2 de master de especialización, que dificulta en gran manera la homologación y por lo tanto la movilidad de los estudiantes españoles a universidades europeas, uno de los objetivos que se pretendían con la creación del EEES.

Tampoco se da dicha movilidad dentro del país, pues en el mapa de titulaciones de la universidad española, aparecen en muchos casos las mismas titulaciones, pero sin coincidir en la denominación, ni en las opciones curriculares, ni en el número de créditos en las materias; todo lo cual dificulta la movilidad de los estudiantes entre universidades por la problemática de las convalidaciones.

Proliferó también en exceso el número de universidades con campus mal planificados, en los que se duplican y triplican titulaciones; los programas contienen materias excesivamente compartimentadas dirigidas por distintos profesores con metodologías distintas; la optatividad en muchos casos es excesiva y poco relevante de acuerdo a la titulación.

En fin, la situación es bastante caótica y como resultado, tenemos graduados con más o menos conocimientos y con un título, y... estos titulados, ¿son creativos, autónomos? ¿resuelven por sí mismos? ¿han aprendido que tienen 
que seguir aprendiendo? ¿han adquirido esas competencias para su desarrollo personal y profesional, actitudes de confianza en sí mismos, sentido crítico, iniciativa personal, curiosidad, interés?

Lo que sabemos, es que pasan a engrosar la lista del paro, o deciden emigrar u opositar a los distintos cuerpos de la Función Pública (muy restrictivos en la actualidad). La tasa de paro alcanza el $12,4 \%$ (2012) frente al $5,2 \%$ en la UE, según el informe anual elaborado por la fundación CYD que evalúa la contribución de las universidades al desarrollo (CYD, 2013). Los egresados presentan una baja predisposición a emprender: Solo 2 de cada 20 graduados trabaja por cuenta propia a los tres años de acabar la carrera (CYD, 2013). Casi el $70 \%$ de los jóvenes españoles está dispuesto a emigrar al no encontrar salida; y eso que nunca España había tenido una juventud con un porcentaje tan alto de estudios superiores: El $39 \%$ de los españoles entre 25 y 35 años tiene un título universitario (CYD, 2013; La Vanguardia, 2013).

Otra posibilidad es opositar, ya que la investigación en este país, fue, es y será muy minoritaria, sacrificada y vocacional. La investigación nunca estuvo lo suficientemente valorada y menos pagada; y eso que, a ella se le deben en gran parte los avances de la ciencia, pues el $80 \%$ de la investigación se realiza en las universidades.

El maestro/bibliotecario o bibliotecario escolar, salvo que sea contratado en algún centro privado, ha de opositar. ¡Primera alarma! En La Orden ECD/713/2013, por la que se convoca el procedimiento selectivo de ingreso al Cuerpo de Maestros, el temario que ha de regir en el procedimiento, figura en el anexo 1 de la orden de 9 de septiembre de 1993, muy actualizado (BOE 103, 2013). Si a esto añadimos el procedimiento de preparación del proceso basado en la memorización de temarios elaborados y publicados por editoriales específicas y academias que se dedican incluso a la elaboración de la programación y las unidades didácticas. El resultado, es un profesional no muy creativo, ni con mucho sentido crítico, ni iniciativa, ni innovador.

¿Cómo es y en qué momento se encuentra el cuerpo de maestros en su entorno escolar? EI maestro accede al magisterio, con el aprendizaje que trae de la facultad, basado en un sistema totalmente tradicional... En la escuela tiene que sujetarse a un programa establecido por las consejerías de educación de las comunidades, atenerse a los libros de texto de la editorial que el centro decide y aplicar los sistemas de evaluación reglados.
Los maestros/as de cierta edad también sufren cansancio, estrés y son conscientes de que necesitan formación para actualizar sus metodologías de enseñanza que contribuyan a mejorar el aprendizaje de los alumnos/as.

En conclusión, tenemos mayoritariamente tanto en la escuela como en la universidad un modelo de enseñanza enlatado y encorsetado. Aquí están las contradicciones a las que nos hemos referido, entre las declaraciones de intenciones y la cruda realidad.

\section{4. ¿Cómo son los niños/as del siglo XXI?}

Los niños/as comienzan su desarrollo desde que nacen de acuerdo a su natural reloj biológico y genético, pero la respuesta madurativa, el desarrollo de su cerebro y facultades emocionales, están condicionadas por los entornos en los que se crían, viven y se educan. No cabe duda que en sus primeros años presentan las mejores condiciones para asimilar pautas de aprendizaje y adquirir las actitudes que promuevan sus mayores.

La infancia del siglo $X X I$, nace muy despierta y los estímulos externos que recibe hacen que tenga características diferentes a las de generaciones anteriores. En primer lugar, estos niños nacen en un mundo robotizado: casi todas las acciones habituales de la vida cotidiana se resuelven pulsando botones; su realidad es un entorno automatizado. Sin embargo, tienen acceso a muchas más cosas que les estimulan y favorecen el desarrollo de la inteligencia desde cortas edades. Presentan avidez por aprender y hacer, un amplio desarrollo verbal y un alto nivel de energía.

Los medios en los que se mueven mayoritariamente son la televisión y los distintos dispositivos electrónicos —ordenadores, videojuegos, móviles-. Son tremendamente ágiles en su manejo y en comunicarse a través de la redes, lo cual les permite absorber información de todo tipo y comunicarse con el mundo sin importar la raza o nacionalidad. Por ello, les denominamos nativos digitales y ciudadanos del mundo.

Estas herramientas van a ser útiles en el mundo competitivo en el que vivimos, permiten desarrollar patrones de atención diferente y un pensamiento crítico mucho más ágil y agudo, porque pueden aprenden a pensar y analizar la información e ir directamente a la que les interesa. El desarrollo de estas habilidades les va a permitir ser mucho más eficientes en la investigación y el análisis; y adquirir competencia psicolingúisticas para la resolución de problemas 
tanto personales como profesionales en el menor tiempo y con mayor acierto (Acevedo, 2005).

Este es su mundo y su potencial positivo; pero son niños y es imprescindible que exista orientación, patrones de conducta y valores, responsabilidad de padres y educadores. Por lo tanto, también hay que tener en cuenta los aspectos negativos de la realidad. En sociedades desarrolladas, dice Robert Shaw, los niños muestran tendencias egoístas, por crecer en ambientes superprotegidos, lo que quizás se pueda deber al aumento de la edad media de la maternidad/paternidad -en España 33 años en 2011) — que se les da gusto y son más dependientes y menos autónomos, mas sedentarios y desconocedores del autocontrol y autodisciplina (Shaw; 2003; Acevedo, 2005)

Se está detectando un aumento notable de niños con dificultades de aprendizaje, de fracaso escolar - la tasa de abandono prematuro en España está entre el $25 \%$ y el $30 \%$, el $23,7 \%$ de los jóvenes entre 15 y 29 años ni estudia ni trabaja, y en el informe Pisa España está 12 puntos por debajo de Europa-, y proliferan los desórdenes emocionales y de comportamiento. Los ordenadores y demás dispositivos también están contribuyendo al desarrollo de actitudes individualistas y de aislamiento, aunque se estén comunicando con el mundo en la red. Han decrecido los niveles de atención, siendo más cortos, dispersos y apastillados.

Esta generación no es ni mejor ni peor, es diferente; y es importante que padres y educadores analicen estos cambios, porque no podrán llevar las riendas de su educación si se desconocen sus necesidades, si no se establecen estrategias de comunicación, vigilancia, pautas de disciplina, firmeza, organización, ejemplo, recuperación de valores, todo ello, creando ambientes tanto familiares como escolares positivos, pese a las dificultades. El desánimo, la parálisis y el espíritu negativo, se transmite con efecto de contagio a una generación que ha de contar con los ingredientes contrarios para construir el futuro mirando hacia adelante con entusiasmo y seguridad (Ibídem, 2005):

Los maestros deben darse cuenta de que no es que estos alumnos tengan problemas de aprendizaje sino que aprenden de manera distinta. Ellos demandan el porqué de todo y el significado de lo que les enseñan, pues quieren conectarse con la realidad. Por eso las clases deben ser innovadoras y llenas de actividades prácticas y multisensoriales. Estos niños y jóvenes necesitan retroalimentación sobre su progreso permanentemente. [...] Los nuevos estudios sobre el cerebro y los problemas de aprendizaje son claros en afirmar la importancia de volver la mirada sobre el individuo y partir de ahí brindar una enseñanza óptima.

\section{El maestro bibliotecario, ¿puede llegar a ser una figura significativa?}

La biblioteca escolar y la figura del maestrobibliotecario pueden jugar un papel transcendental en la conjugación y aprovechamientos del potencial natural intelectual con que cuentan los niños/as y sus habilidades tecnológicas, haciendo de guía-orientador y propulsando un cambio en los sistemas y metodologías de enseñanza, apoyando al resto del profesorado.

El nuevo concepto de biblioteca escolar sin muros contribuye a crear el marco de escuela abierta. Esta biblioteca se constituye en un sistema de múltiples medios y soportes de información, siendo la mejor plataforma para recibir los avances y cambios que el medio genera continuamente, facilitando el aprendizaje independiente. Por ello, el bibliotecario escolar y/o maestro-bibliotecario debe colaborar en el proceso del diseño curricular, contribuyendo a la selección de contenidos, a la identificación de recursos y materiales, y ser agente activo en el desarrollo de los programas diseñados. De esta manera, cumplirá la importante función de asesoramiento y de soporte a la escuela; no solo a los alumnos, sino también a los profesores, colaborando con ellos en las tareas docentes. El bibliotecario escolar genera una nueva dinámica de trabajo promocionando una metodología basada en la investigación del alumno guiada por el profesor; y la biblioteca pasa de ser un mero complemento a estar integrada y ser parte esencial y constitutiva del método (Ibídem, 1997).

El bibliotecario escolar será el médium para extender este concepto de biblioteca en la escuela y estar embebido en la organización escolar. Como sabemos no hay vías de momento para que las escuelas cuenten con un titulado en esta especialidad.

Por ello, vemos la posibilidad de apostar por la figura del maestro-bibliotecario, con la titulación de maestro y la formación complementaria en biblioteca escolar con las mismas funciones que describíamos anteriormente, con la ventaja de que ya está de por sí integrado en el cuerpo docente y se constituiría en pieza clave para integrar la biblioteca en la actividad docente, con el rol de especialista de la información y tratamiento de la misma, como formadorprofesor, como asesor de la enseñanzaaprendizaje y como dinamizador de la biblioteca en todo el entorno escolar. 


\section{Propuesta de un programa para la formación del maestro bibliotecario}

Es evidente que un maestro bibliotecario, no va a contar con la misma formación que un titulado especializado en el área de la biblioteconomía, aunque la biblioteca escolar sería solamente una parcela de su formación. Tampoco existe ánimo de acaparar este nicho profesional, sino que la intención es poder evitar que la biblioteca escolar quede diluida o suplantada por la tecnología, perdiendo su esencia.

La opción del maestro bibliotecario es una oportunidad que se puede aprovechar desde las facultades de formación de profesorado, ofertando este itinerario como mención o como máster.

Algunas facultades y escuelas de documentación y biblioteconomía ofrecen master en bibliotecas escolares; $y$, si son abiertos, también son una buena opción para que los maestros puedan adquirir una especialización.

De todas formas, la posibilidad de formar al bibliotecario dentro de la propia titulación de maestro la encontramos muy positiva. La nueva titulación de grado en maestro solamente tiene dos opciones: infantil y primaria. Su formación es generalista, además de ir acompañada de una fuerte carga docente en psicología infantil y de formación en habilidades pedagógicas. Si le añadieramos la formación específica y se lograra que asimilaran el concepto de biblioteca escolar como la hemos definido, podría llegar a ser un elemento estratégico en la escuela.

La propuesta del itinerario formativo del maestro-bibliotecario en la Facultad de Formación de Profesorado de Lugo (USC) fue aprobada por la Comisión de Titulaciones de la Facultad y de la Universidad, recogida en la Memoria para la Verificación del título de grado en Maestro de Educación Primaria y aprobada por la Agencia de Calidad del Sistema Universitario Gallego, ACSUG (USC, 2009). Dicha propuesta se plasmó en un documento donde previamente se define el modelo formativo, se justifica la importancia de su existencia y se diseña el programa docente.

\section{El modelo}

Una biblioteca escolar se concibe como una herramienta educativa y un profesional que facilite al estudiante el aprendizaje, la adquisición de competencias informacionales, hábitos lectores, actitudes creativas y habilidades intelectuales y comunicativas, además de constituirse en el centro neurálgico de referencia para el profesorado y toda la comunidad escolar.

\subsection{Justificación}

La sociedad en la que vivimos exige personas capaces de utilizar y evaluar la información en sus diferentes formatos. La multialfabetización se ha convertido en un requisito indispensable para lograr ciudadanos activos. Es fundamental que este proceso de aprendizaje comience en edades tempranas y continúe a lo largo de la vida.

Se tiene que hacer la reflexión sobre la necesidad de mejorar estas habilidades y el papel de los maestros como gestores de información en esta tarea. Es clave la comprensión por parte de estudiantes, maestros y maestros-bibliotecarios de que las habilidades en información y comunicación son fundamentales para que el estudiante sea guía de su propio aprendizaje.

Es importante también entender el marco conceptual que configura a la biblioteca escolar como centro de recursos para la enseñanza y el aprendizaje (CREA). asociado a las nuevas tecnologías; valorar su misión, sus funciones y posibilidades reales de desarrollo en los centros educativos; que el funcionamiento de una biblioteca en la escuela sea una herramienta útil para la consecución del currículum y la realización práctica educativa.

\subsection{Organización docente de la mención cualificadora biblioteca escolar y programa de formación del maestro-bibliotecario}

Hay que tener en cuenta, el marco legislativo en el que se encuadra esta mención, sus objetivos y la organización de la enseñanza: área, materias, actividades, contenidos, metodología, competencias y evaluación.

\subsection{Marco legislativo-normativo}

Del Ministerio de Educación y Ciencia proceden las Actuaciones para la mejora de las bibliotecas escolares. La Ley Orgánica 2/2006, de 3 de mayo, de Educación, expone la importancia del fomento de la lectura y la mejora de las bibliotecas de los centros escolares; e incluye, dentro de los fines de la educación, la atención prioritaria que los poderes públicos deben prestar a este factor educativo; y menciona expresamente que las bibliotecas escolares contribuirán a fomentar la lectura y a que los alumnos accedan a la información y otros recursos para el aprendizaje disponibles en las mismas. (BOE n. 2006)

Estos objetivos están íntimamente asociados al desarrollo de las competencias básicas estable- 
cidas en el Real Decreto 1513/2006, de 7 de diciembre (BOE, 293) por el que se establecen las enseñanzas mínimas respectivamente de la educación infantil, primaria y la educación secundaria obligatoria. En particular la biblioteca escolar es un elemento imprescindible para el adecuado logro de las competencias en comunicación lingüística, para aprender a aprender, en el tratamiento de la información y la autonomía e iniciativa personal, y es también un recurso muy útil para la adquisición del resto de las competencias básicas. (BOE 293, 2006)

En la Orden ECl/3857/2007 de 27 de diciembre, se establecen los requisitos para la verificación de los títulos universitarios oficiales que habiliten para el ejercicio de la profesión de Maestro en Educación Primaria (BOE 312, 2007). En su anexo -concretamente en el apartado 5 de planificación de las enseñanzas y menciones cualificadoras - se contemplan entre 30 y 60 créditos europeos, adecuadas a los objetivos, ciclos y áreas de la educación primaria y asociadas a las competencias educativas, tales como: biblioteca escolar, las TIC y la educación de personas adultas.

\subsection{Objetivo general}

Su objetivo general es formar personal especializado para dar respuesta a las necesidades detectadas en el ámbito educativo sobre la biblioteca escolar como escenario de aprendizaje $y$ al mismo tiempo sean agentes dinamizadores y facilitadores de competencias y generadores de actitudes en cuanto a participación, cooperación e interacción

\subsection{Objetivos concretos}

Los objetivos concretos son los siguientes_

- Formar maestros capacitados para organizar y gestionar una biblioteca escolar.

- Formar a estos profesionales como formadores en la adquisición de competencias transversales, informacionales y tecnológicas.

- Dar a conocer el valor de las bibliotecas escolares como recurso didáctico.

- Dar a conocer las actividades para dinamizar las bibliotecas.

- Proponer una visión moderna de las técnicas de documentación y uso de las nuevas tecnologías en relación con el acceso a la información.

\subsection{Organización de la enseñanza}

La mención se distribuye en cuatro materias optativas que se ofertan en $4^{\circ}$ curso de grado de la titulación. Cada una tiene una carga de 4,5 créditos; el practicum, 3 créditos; y el proyecto, 3 créditos igualmente. La mención Biblioteca escolar: nuevo escenario para el apoyo a la docencia y aprendizaje se adscribe al área de Didáctica y Organización Escolar.

El modelo docente o programa de formación se ha diseñado teniendo en cuanta los objetivos propuestos y el modelo de biblioteca escolar y profesional que se quiere alcanzar. Con esa orientación se decidieron los contenidos y las competencias. En cuanto a la actividad y metodología de enseñanza, se propone llevarla a cabo en formato de aprender haciendo, tomando cada una de las materias como un proyecto. El objetivo es que los futuros maestros-bibliotecarios aprendan a trabajar creando, construyendo el conocimiento de la materia, con su parte autónoma, debatiendo y tomando decisiones en grupo, y llegar a la exposición general del conjunto.

Se propone el mismo modelo, competencias y criterios de evaluación para todas las materias, solamente las diferencia la denominación y los contenidos.

\subsubsection{Materias y contenidos}

Cada una de las materias se organiza en 6 módulos temáticos

\section{Organización y gestión de la biblioteca escolar}

1. Concepto, objetivos y funciones de la biblioteca escolar del siglo XXI.

2. Instalaciones y equipamiento en una biblioteca concebida como espacio sin muros y la integración de las Nuevas tecnologías de la información y la comunicación.

3. Las colecciones en propiedad y los distintos modelos de acceso a la información.

4. Procesos técnicos: adquisición, tratamiento y difusión de la información.

5. Servicios de la biblioteca: préstamo, información y formación.

6. Los recursos humanos de la biblioteca escolar: competencias y rol del maestro bibliotecario.

Alfabetización Informacional desde la biblioteca escolar

1. Formulación general y fundamentación de la Alfabetización informacional (Al). 
2. Análisis de entornos y destinatarios de ALFIN.

3. El maestro/bibliotecario formador en ALFIN.

4. Programas nacionales e internacionales ALFIN.

5. Implantación y difusión de ALFIN.

6. Evaluación de habilidades informacionales.

Biblioteca escolar como unidad de apoyo a la docencia y aprendizaje

1. La biblioteca escolar como centro de recursos de apoyo a la docencia y al aprendizaje.

2. El maestro bibliotecario como diseñador y recolector de materiales educativos. Los repositorios de materiales docentes.

3. La biblioteca como factoría de materiales de apoyo a la docencia y objetos de aprendizaje en el área de las Ciencias sociales y conocimiento del medio: recursos y herramientas.

4. La biblioteca como factoría de materiales de apoyo a la docencia y objetos de aprendizaje en el área de las ciencias experimentales y la educación medio ambiental: recursos y herramientas.

5. La biblioteca como factoría de materiales de apoyo a la docencia y objetos de aprendizaje en el are de la sociología, la diversidad cultural, etnológica y la formación en valores: recursos y herramientas.

6. La biblioteca como factoría de materiales de apoyo a la docencia y objetos de aprendizaje en lenguas extranjeras: recursos y herramientas.

\section{Dinamización de la biblioteca escolar}

1. La biblioteca como agente promotor de la lectura: la librería infantil y juvenil, talleres de lectura comentada, exposiciones.

2. La biblioteca como agente promotor de la creación: las exposiciones plásticas, creación musical, creación literaria, dramatización.

3. La biblioteca como agente dinamizador de la cultura en la escuela: la prensa escolar, la radio escolar.

4. La biblioteca escolar como espacio socializador: Biblioteca 2.0.

5. La biblioteca escolar como espacio de comunicación: herramientas de comunicación (wikis, blog...).
6. La biblioteca como agente responsable en la escuela de la educación de los jóvenes en el buen uso de Internet

\subsubsection{Competencias}

Se seleccionan entre las competencias generales y básicas del Ministerio, las específicas de la materia y las transversales reconocidas por la USC. Las competencias generales asignadas fueron:

- G.2: Aprenden a diseñar, planificar y evaluar proyectos individualmente y en colaboración.

- G.5: Fomentan la convivencia en el aula, liderando, estimulando, esforzándose.

- G.10: Reflexionan sobre las practica de aula y muestran capacidad de innovación.

las competencias específicas:

- E-1: Capacidad de planificación y gestión de la materia.

- E-2: Capacidad para identificar contenidos y materiales apropiados de la materia.

las competencias básicas:

- B.1: Que los estudiantes hayan demostrado poseer y comprender conocimientos en el área de estudio.

- B.2: Que los estudiantes sepan aplicar sus conocimientos, sepan defender argumentos y resolver problemas dentro de su área de estudio.

y las competencias transversales de la universidad

- T.1: Lenguas extranjeras

- T.2: Lengua gallega

- T.3: Competencias informáticas e informacionales $\mathrm{Cl} 2$

\subsubsection{Actividad docente}

Corresponde a la organización de la docencia según el crédito horario y la nueva distribución de la enseñanza. La secuencia es básicamente la siguiente:

- Actividad en gran grupo, expositivo (presencial)... Exposición del plan de trabajo. Formación de grupos de trabajo. Exposición de los puntos fundamentales de cada tema. Establecimiento de pautas de trabajo. Propuesta de información. Herramientas de comunicación...

- Trabajo autónomo del alumno. 
- Diseño de los proyectos relativos a cada tema: planificación y proceso. Búsqueda de información relativa al proyecto. Análisis, síntesis y obtención de resultados. Diario de trabajo. Explicitacion del proyecto.

- Actividad en grupo mediano, interactivo (presencial y virtual). Coordinación del proyecto. Reconducir líneas equivocadas. Estimular el debate. Aplicar sistemas comparativos. Control compartido del desarrollo del proyecto...

- Trabajo autónomo del alumno.

- Plantear el diseño, los objetivos y el proceso. Analizar y comparar distintas iniciativas.

- Adaptar la selección al proyecto propuesto. Proporcionar sistemas de evaluación. Defensa del proyecto.

- Actividad pequeño grupo, tutoría (presencial y virtual). Tutorías grupales para resolución de problemas y conflictos. Tutorías individuales a demanda. Comunicación presencial y telemática.

- Trabajo autónomo

- Desarrollo individual y práctico de un aspecto de cada tema. Documentar la actividad. Redacción final del trabajo

\subsubsection{Metodología}

El planteamiento de la materia y la metodología que se pretende utilizar se orienta a fomentar en el alumno la capacidad de investigación autónoma así como la gestión de conocimiento en colaboración y en torno al concepto de proyecto. El profesor actuará como tutor, asesor y reconductor con respecto a los contenidos, metodología de trabajo y actitudes, con el objetivo de que el resultado final en el aprendizaje del alumno sea una visión clara compartida y debatida sobre la temática de cada una de las materias:

- Visión clara compartida y debatida sobre la gestión de una biblioteca escolar en el siglo $\mathrm{XXI}$ y los distintos aspectos a tener en cuenta en la organización y administración de la misma. El proyecto de gran grupo consistirá en el diseño de una biblioteca escolar que se irá construyendo con las aportaciones de todos los grupos y las hojas de procedimiento que aportarán los trabajos individuales.

- Alfabetización Informacional. En gran grupo se debatirán las distintas alternativas que circulan a nivel nacional e internacional y la importancia que cada una ha tenido en el país de origen y en su entorno educativo. Los distintos grupos realizarán trabajos es- pecíficos de diseño de programas ALFIN multidisciplinares, específicos, y encuestas de evaluación de habilidades que experimentarán con colectivos concretos. Individualmente podrán elaborar un trabajo de contenido libre utilizando y trabajando con cada uno de los pilares ALFIN.

- Escenario de aprendizaje y factoría recolectora y productora de materiales educativos. El proyecto final consistirá en el diseño y arquitectura de un repositorio de materiales digitales de todas las áreas temáticas incluidas en el temario. El trabajo grupal se centrará en la elaboración de cada una de las partes del repositorio e individuamente trabajará en la recuperación y diseño de algún material concreto de cada una de las áreas.

- Rol de la biblioteca escolar como agente dinamizador de la cultura, espacio socializador y de comunicación tanto física como digital. El proyecto se orientará a la creación de un espacio que englobe cada una de las actividades propuestas en el temario que irán desarrollando cada uno de los grupos de trabajo con las aportaciones de pequeños proyectos, programas e iniciativas novedosas de cada uno de los alumnos.

\subsubsection{El prácticum y el trabajo de fin de grado}

Se procurará buscar centros escolares que tengan algún desarrollo de biblioteca escolar o estén dispuestos a que se pueda llevar a cabo alguna actividad práctica o pequeño proyecto relacionado con alguno de los temas de la mención. Así mismo, el TFG o proyecto fin de grado, consistirá en una memoria de planificación sobre algún aspecto, actividad, buena práctica, etc., de los bloques temáticos de la mención, que pueda permitir evaluar los conocimientos y las competencias adquiridas.

\subsubsection{Profesorado}

La propuesta de esta mención fue iniciativa de la Dirección de la biblioteca de la Facultad apoyada por el Decanato. Se estableció el compromiso de que la coordinación de la mención recayese en la biblioteca, trabajando con el resto del profesorado implicado de forma colaborativa, para conseguir que la docencia se desarrollase coordinadamente. Las materias se montarían a su vez en el aula virtual como parte de la metodología de trabajo.

\subsubsection{Evaluación}

La evaluación sería continua y teniendo en cuenta todos los parámetros comprendidos en 
la competencias definidas. La valoración se haría a nivel individual, grupal y en conjunto.

\subsubsection{Calidad}

El proceso de aseguramiento de la calidad incluye la elaboración y evaluación de las guías docentes y la confección de una memoria final de autoevaluación, e incorporación en el Sistema de Gestión de Calidad Interna del Centro (SGCIC).

\section{Conclusión}

Conoces la situación que te rodea en parcelas, pero, cuando te propones engarzarlas como se ha tratado de hacer en este artículo -que, además de tener el objetivo de exponer una propuesta, significó un ejercicio de reflexión haciendo el recorrido por los ambientes de cada una de los colectivos afectados-, sacas como conclusión que el panorama es gris y poco alentador, con mucho que organizar y que reconducir, una labor titánica.

De lo que no dudamos es que el camino hay que comenzar a abrirlo desde el principio, desde las edades más tempranas. El maestro, de por sí, siempre fue una figura muy importante en todo el recorrido educativo; y el magisterio no estuvo suficientemente valorado en este país. Son los maestros quienes empiezan a moldear las capacidades intelectuales que han de tener los jóvenes y los adultos del futuro. Por ello, y teniendo en cuanta la situación actual de la enseñanza y el mundo que nos rodea, vemos tan importante la figura de un profesional que conjugue conocimiento, pedagogía y formación bibliotecaria para comprender el papel que debe jugar en la escuela y en la biblioteca escolar.

Compartimos la opinión de Gómez Hernández con respecto a que las bibliotecas escolares y sus profesionales: Son un elemento clave para la función transformadora que se tiene que dar en la educación, una vía para resolver muchas carencias que conducen al fracaso escolar, a los pobres resultados escolares, posibilitando la interacción y el aprendizaje autónomo y compartido (Gómez-Hernandez, 2013):

Posiblemente sea una utopía que tiene que ver con lo que querríamos conseguir, pero soñar es también pragmático, pues sin sueños no se puede mejorar la realidad, sino que meramente se reproduce.

Quienes queremos que sea posible somos personas implicadas, convencidas, que seguimos trabajando, escribiendo, difundiendo, denunciando y aportando soluciones.
Los que aseguran que es imposible, no deberían interrumpir a los que estamos intentándolo. (Thomas Alva Edison)

\section{Referencias}

Acevedo, Anni Rehbeing (2005). Casos y cosas: la realidad de los niños y jóvenes de hoy. Bogotá: Norma, http://books.google.es/books?id=90U3JyL3aPsC\&pg=P A1\&lpg $=P A 7 \&$ ots $=$ CZmWPwZP\&dq $=$ casos $+y+$ cosas +1 a+realidad+de+los+ni\%C3\%B1os+y+j\%C3\%B3venes $+d$ e+hoy\&hl=es 11-05-2013).

Area Moreira, M. (2005). Internet en la docencia universitaria: webs docentes y aulas virtuales.Tenerife: Universidad de La Laguna, Departamento de didáctica e investigación educativa. http://webpages.ull.es/users/manarea/ guiadidacticawebs.pdf (19-05-2013).

Bueno Monreal, María (1997). Aportaciones metodológicas y curriculares de la biblioteca escolar en el desempeño profesional del profesor: el bibliotecario escolar y su formación. // Revista electrónica Interuniversitaria de Formación de Profesorado. 1:0. http://www.uva.es/aufo p/publica/actas/viii/edprima.htm (19-05-2013)

Durban, Gloria (2009). El qué y para qué de la biblioteca escolar: Reflexión en torno a su implementación como recurso educativo útil. // Bibliotecaescolar.info.h ttp://www.bibliotecaescolar.info/varis/biblioteca_impleme ntacion.pdf (19-05-2013).

Fundación Conocimiento y Desarrollo, CYD (2013). Informes CYD. http://www.fundacioncyd.org/ (19-05-2013).

Gómez Hernández, Jose Antonio (2010). Las bibliotecas escolares en España ante una nueva década. // Anuario ThinkEpi: Análisis de tendencias en información y documentación. 4 (2010) p. 94-102.

Gómez-Hernández, José Antonio (2013). Unir educación y biblioteca: la evolución de un reto permanente. // El profesional de la Información. 22:2, 101

Ley Orgánica 10/2002, de 23 de diciembre de Calidad de la Educación. // Boletín Oficial del Estado de 23 de diciembre. 207, 45188.

Ley Organica 2/2006, de 3 de mayo, de Educación. // Boletín Oficial del Estado de 3 de mayo. 307, 17158 ss.

Ley Organica 11/1983 de 25 de agosto, de Reforma Universitaria. // Boletín Oficial del Estado. 209, 24034 ss.

Ley Orgánica 6/2001, de 21 de diciembre, de Universidades. Boletín Oficial del Estado. 307, 49400 ss.

Ley Orgánica 4/2007, de 12 de abril, por la que se modifica la Ley Orgánica 6/2001, de 21 de diciembre, de Universidades. // Boletín Oficial del Estado. 89, 16241 ss.

Marzal, Miguel Ángel; Sellers de los Rios, Nieves (2011). Instrumentos de desarrollo de competencias para un programa de alfabetización en información en bibliotecas escolares. // Revista General de Información y Documentación. 21, 53-78. http://revistas.ucm.es/index. php/RGID/article/view/53-78 (28-08-2013).

Orden ECI/3857/2007, de 27 de diciembre, por la que se establecen los requisitos para la verificación de los títulos universitarios oficiales que habiliten para el ejercicio de la profesión de Maestro en Educación Prima. // Boletín Oficial del estado. 312 ( 27 de diciembre) $53747 \mathrm{ss}$

Orden ECD/713/2013, de 23 de abril, por la que se convoca procedimiento selectivo de ingreso al Cuerpo de Maestros para plazas del ámbito de gestión territorial del Ministerio de Educación, Cultura y Deporte y procedimiento de adquisición de nueva especialidad para los funcionarios de carrera del citado cuerpo. // Boletín Oficial del Estado de 23 de abril. 103, 32611 ss. 
El Paro se duplica entre los universitarios. (2013). // La Vanguardia. (8 de mayo 2013). http://www.lavanguardia .com/.../el-paro-se-duplica-entre-los-universitarios (1005-2013)

Real Decreto 1513/2006, de 7 de diciembre, por el que se establecen las enseñanzas mínimas de la Educación primaria. // Boletín Oficial del estado del 7 de diciembre. 293, 43053 ss.

Universidad de Santiago de Compostela (2009). Memoria para la verificación del título de grado en maestro/a de educación primaria. http://www.usc.es/export/sites/de fault/gl/centros/ffp/descargas/Grao_Mestre_Educacion_ Primaria.pdf (19-05-2013).
Varela-Prado, Carmen. Adquisición de competencias en elearning: competencias en información una responsabilidad compartida docencia/biblioteca. IBERSID: revista de sistemas de información y documentación, 2010, vol. 2009, pp. 303-312. http://eprints.rclis.org/13190/ (19-052013).

Enviado: 2013-05-19. Segunda versión: 2013-08-28 Aceptado: 2013-09-02. 